PROTEIN DESIGN

\section{We built this protein}

Nat. Struct. Mol. Biol. doi:10.1038/nsmb.2938

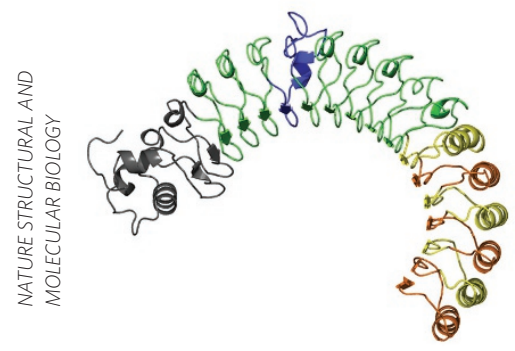

Repeat proteins are composed of tandem arrays of smaller protein modules, and many of these proteins have a concave surface that is used to bind other proteins. For this reason, repeat proteins have been engineered-for example, by changing amino acid residues at their curved binding surfaces or by altering the number of or the sequence of the repeated modules-to bind non-native protein targets. However, a general approach to systematically alter the shape and curvature of the designed protein has not been reported. Park et al. identified four naturally occurring leucine-rich repeat (LRR) modules that had well-defined shapes, suggesting that they could be used as potential 'building blocks' to construct larger, non-natural proteins with predictable three-dimensional structures. The authors optimized the sequences of the LRR modules using a previously published Rosetta repeat-protein idealization method to ensure that the modules would be stable and behave predictably in vitro. They then synthesized proteins that had five to seven copies of each of the idealized building block sequences and solved the X-ray crystal structures of these polyproteins: two of the building blocks formed solenoid-like structures, and the other two were highly curved. The authors also designed five 'junction modules', which could be used to connect the building blocks in various orientations, and a 'wedge' module that would further alter the curvature of the designed protein. Using these modules, the authors constructed four larger proteins-composed of 10-19 components-and demonstrated that they had well-defined CD spectra and high thermal stability and unfolded in a cooperative manner. X-ray crystal structures of two of them closely matched the models, indicating that these modules could reliably generate larger proteins with predictable structures and different overall shapes and curvatures. The authors estimated that using just 12 components, one could generate nearly 19,000 different macromolecules that could be further engineered using computational protein design or directed evolution to selectively bind specific target proteins. JMF

RNA EPIGENETICS

\section{$\mathrm{m}^{6} \mathrm{~A}$ partial differential \\ Science doi:10.1126/science.1261417}

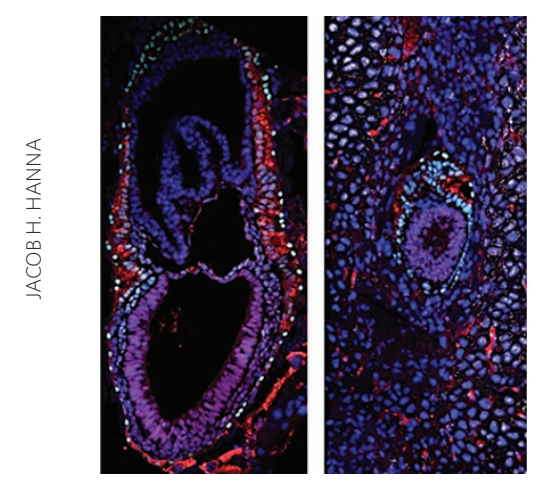

Naive pluripotent cells can be stimulated to transition into a primed state that readies them for further differentiation. Yet, the molecular bases for maintenance of pluripotent states and the signaling pathways that govern the transition between them remain relatively unexplored. To gain further insight into these processes, Geula et al. screened a library of siRNAs targeting genes with potential roles in pluripotency reprogramming of mouse embryonic stem cells (mESCs). A number of epigenetic regulators, including proteins in the polycomb complex, were identified along with Mettl3, a protein that collaborates with Mettl14 as part of the machinery that installs $N^{6}$-methyladenosine $\left(\mathrm{m}^{6} \mathrm{~A}\right)$ modifications in mRNA. To explore the function of Mettl3 and $\mathrm{m}^{6} \mathrm{~A}$ modification in pluripotency and differentiation, the authors generated a targeted knockout of Mettl3 in mice and derived ESCs from these animals. Knockout mESCs sustained a naive pluripotent state in which pathways involved in priming and differentiation are blunted. MS analysis revealed that mRNAs from the knockout mESCs have fewer $\mathrm{m}^{6} \mathrm{~A}$ marks relative to wild-type ESCs, and RNA-seq profiling of $\mathrm{m}^{6} \mathrm{~A}$ modifications across developmental stages showed that Mettl 3 and $\mathrm{m}^{6} \mathrm{~A}$ depletion directly enhanced the stability of mRNAs for genes that maintain naive pluripotency. Ribosome profiling analysis confirmed that the resultant elevated protein levels were not directly related to the depletion in $\mathrm{m}^{6} \mathrm{~A}$ methylation on mRNA transcripts. Taken together, the results support the idea that $\mathrm{m}^{6} \mathrm{~A}$ modification of mRNA has an important regulatory role in the orderly transition between naive and primed pluripotent states and highlight the need for a deeper understanding of the machineries that write, erase and read these RNA epigenetic marks.

LIPID-PROTEIN INTERACTIONS

\section{Three sites in PTEN \\ J. Biol. Chem. 290, 1592-1606 (2015)}

PTEN is a lipid phosphatase that acts as a tumor suppressor through its action on the PI3K/Akt signaling pathway. $\mathrm{PI}(3,4,5)$ $\mathrm{P}_{2}$ (phosphatidylinositol 4,5 bisphosphate) is the most preferred PTEN substrate, but $\mathrm{PI}(3,4) \mathrm{P}_{2}$ and $\mathrm{PI}(3) \mathrm{P}$ can also be dephosphorylated by PTEN. Another $\mathrm{PIP}_{2}$, $\mathrm{PI}(4,5) \mathrm{P}_{2}$, potentiates PTEN activity towards $\mathrm{PI}(3,4) \mathrm{P}_{2}$ and $\mathrm{PI}(3) \mathrm{P}$ by enhancing the enzyme's binding to membranes. $\mathrm{PI}(4,5)$ $\mathrm{P}_{2}$ kinetic activation studies, structural studies and molecular dynamics simulations have implicated an N-terminal PTEN loop as the $\mathrm{PI}(4,5) \mathrm{P}_{2}$ binding site. Besides this site and the substrate-binding site, an amphiphile-binding site (known as AHS) defines a third functionally distinct region of the catalytic domain of PTEN. To locate more specifically the $\mathrm{PI}(4,5) \mathrm{P}_{2}$ binding site and the regulation by $\mathrm{PI}(4,5) \mathrm{P}_{2}$, Wei et al. characterized the binding and activity of a series of phosphatidylinositols to PTEN using high-resolution field cycling ${ }^{31} \mathrm{P}$ NMR spectroscopy on spin-labeled protein along with enzyme kinetics. They found that $\mathrm{PI}(4,5) \mathrm{P}_{2}$ differentially acts as an inhibitor or activator of PTEN depending on the substrate ( $\mathrm{PI}(3) \mathrm{P}$ or $\mathrm{PI}(3,4) \mathrm{P}_{2}$, respectively). Mutation of Lys13, a residue important in $\mathrm{PI}(4,5) \mathrm{P}_{2}$ activation of PTEN, or Arg47 in the AHS site abrogated the ability of $\mathrm{PI}(4,5) \mathrm{P}_{2}$ to activate PTEN, suggesting that the $\mathrm{PI}(4,5) \mathrm{P}_{2}$ activator site is near the AHS and is distinct from the AHS (which they also found to involve Arg47). The NMR experiments further validated three distinct sites, where $\mathrm{PI}(4,5)$ $\mathrm{P}_{2}$ is clearly not binding the active site, and showed that even when $\mathrm{PI}(4,5) \mathrm{P}_{2}$ is unable to activate PTEN (when Lys13 or Arg47 is mutated), it does still bind the enzyme. The results further suggest that the orientation of $\mathrm{PI}(4,5) \mathrm{P}_{2}$ within $\mathrm{PTEN}$ is very specific, as might be expected, as is its close proximity to the substrate lipid. The authors propose a model where $\mathrm{PI}(4,5) \mathrm{P}_{2}$ enhances anchoring of PTEN to the membrane by limiting flexibility of the N-terminal PTEN domain and acts as an electrostatic anchor for processive catalysis where substrate and product can swiftly enter and exit the active site.

$M B$

Written by Mirella Bucci, Joshua M. Finkelstein,

Catherine Goodman, Grant Miura and

Terry L. Sheppard 